INTERNATIONAL BULLETIN OF BACTERIOLOGICAL NOMENCLATURE AND TAXONOMY

Vol. 15, No. I January I5, 1965

pp. $39-42$

\title{
PROPOSAL FOR RECOGNITION OF NEOTYPES OF BACTERIAL SPECIES AND SUBSPECIES
}

Martinec and Kocur in a monograph include descriptions and historical background of 30 nominal species that have been placed in the genus Erwinia. Physiological characters of 155 strains are included. For some species references in the literature only were available. All are adequately discussed. They propose that two species be recognized in the genus, E. amylovora, with E. amylovora subsp. salicis, and $E$. carotovora. Neotypes are proposed for the three taxa as follows:

E. amylovora (Burrill) Winslow. BS 1114 (ATCC 15580; NCPPB 683)

E. amylovora subsp. salicis (Day) comb. nov. BS 1027 (ATCC I57 12 ; NCPPB 447)

E. carotovora (Jones) Bergey et al. BS 1008 (ATCC 15713; NCPPB 310)

The characters of proposed neotype strains 1114 (1963, 19-20), $1057(1963,39-40)$ and $1008(1963,54-55)$ are listed in Table l. Objections or criticisms of the proposals should be sent to the Chairman of the Judicial Commission or to the Editorial Secretary. The proposals will be submitted to the Judicial Commission six months after the publication of the se proposals under date of January 15, 1965.

Table 1. Characteristics of proposed neotype strains.

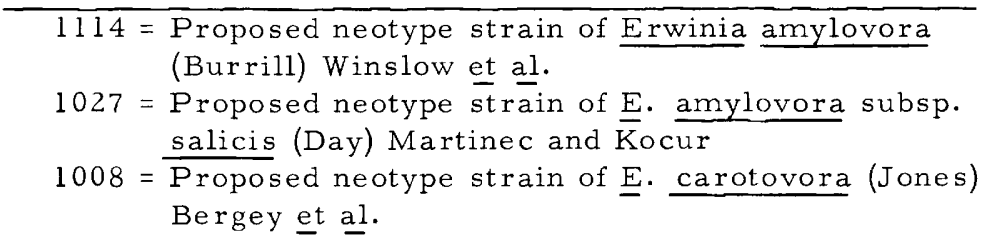

\begin{tabular}{llll}
\hline Test & 1114 & 1027 & 1008 \\
\hline
\end{tabular}

\section{Glucose}

Gas from glucose

Lactose

Sucrose

Maltose

Galactose

$\begin{array}{lll}+ & + & + \\ - & + & + \\ - & - & + \\ + & + & + \\ - & + & - \\ + & + & +\end{array}$


Page 40

INTERNATIONAL BULLETIN

Table 1. (cont.)

\begin{tabular}{|c|c|c|c|}
\hline Test & 1114 & 1027 & 100 \\
\hline Fructose & + & + & + \\
\hline Rhamnose & - & - & + \\
\hline Mannose & + & + & + \\
\hline Inulin & - & - & - \\
\hline Xylose & - & + & + \\
\hline Arabinose & - & - & - \\
\hline Cellobiose & - & - & + \\
\hline Adonitol & - & - & - \\
\hline Sorbitol & + & + & - \\
\hline Glycerol & - & + & + \\
\hline Mannitol & + & + & + \\
\hline Dulcitol & - & - & - \\
\hline Trehalose & + & + & + \\
\hline Raffinose & + & + & + \\
\hline Inositol & - & + & - \\
\hline Esculin & + & + & - \\
\hline Salicin & + & + & + \\
\hline Hydrolysis of starch & - & - & - \\
\hline Starch (gas in 4 days & - & - & - \\
\hline Cellulose & - & - & - \\
\hline Sodium formate & + & + & + \\
\hline Sodium acetate & - & + & + \\
\hline Sodium lactate & + & + & + \\
\hline Sodium succinate & + & + & + \\
\hline Sodium benzoate & - & - & - \\
\hline Sodium tartrate & - & - & - \\
\hline Sodium malonate & - & - & - \\
\hline Sodium citrate & - & + & + \\
\hline Acetylmethylcarbinol & + & + & + \\
\hline Methyl red test & - & + & + \\
\hline Arginine dihydrolase & - & - & - \\
\hline Lysine decarboxylase & - & + & - \\
\hline Ornithine decarboxylase & - & + & - \\
\hline
\end{tabular}


BACTERIOLOGICAL NOMENCLATURE

AND TAXONOMY

Table 1. (cont.)

\begin{tabular}{|c|c|c|c|}
\hline Test & 1114 & 1027 & 1008 \\
\hline Glutamic acid & + & - & - \\
\hline Phenylalanine deaminase & - & - & - \\
\hline Hydrolysis of gelatine & + & + & + \\
\hline Hydrolysis of casein & - & + & + \\
\hline Reduction of methylene blue & - & + & + \\
\hline Reduction of nitrate & - & + & + \\
\hline Reduction of nitrite & - & - & + \\
\hline Ammonia production & - & - & - \\
\hline Hydrogen sulfide & - & - & - \\
\hline Indol & - & - & - \\
\hline Braun's test (KCN) & - & + & + \\
\hline Catalase & + & + & + \\
\hline Pectinase & - & - & + \\
\hline Cytochrome oxidase & - & - & - \\
\hline Lipase & - & - & - \\
\hline Urease & - & - & - \\
\hline Lecithinase & - & - & - \\
\hline Tyrosinase & - & - & - \\
\hline Hemolysis & - & - & - \\
\hline $5 \% \mathrm{NaCl}$ & - & + & - \\
\hline 7. $5 \% \mathrm{NaCl}$ & - & - & - \\
\hline Growth at $37^{\circ} \mathrm{C}$ & + & + & + \\
\hline Penicillin & - & - & - \\
\hline Streptomycin & + & + & + \\
\hline Chloramphenicol & + & - & + \\
\hline Chlortetracyclin & - & - & + \\
\hline Tetracyclin & + & - & + \\
\hline Erythromycin & + & - & - \\
\hline Neomycin & + & + & + \\
\hline Tyrothricin & - & - & - \\
\hline Nystatin & - & - & - \\
\hline Bacitracin & - & - & - \\
\hline
\end{tabular}




\section{REFERENCES}

Martinec, T. and M. Kocur. 1963. Taxonomická studie rodu Erwinia. (A taxonomic study of the genus Erwinia) Folia (Biologia 2) Facultatis Scientarum Naturalium Universitatis Purkynianae Brunensis (Faculty of Natural Sciences of Purkyni University, Brno) $\underline{4}(6): 1-163$. and - 1964. A taxonomic study of Erwinia

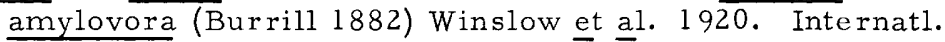
Bull. Bacteriol. Nomen. and Taxon. $\overline{1} 4(1): 5-14$.

R. E. Buchanan, Chairman, Judicial Commission

Erwin F. Lessel, Jr., Editorial Secretary 